8th Alexander Friedmann International Seminar

on Gravitation and Cosmology

International Journal of Modern Physics: Conference Series

Vol. 3 (2011) 276-280

(C) World Scientific Publishing Company

DOI: 10.1142/S201019451100136X

\title{
SEARCHING COSMOLOGICAL MODELS FROM THE PHENOMENOLOGY OF LARGE ACCELERATORS
}

\author{
RENATO KLIPPERT \\ Department of Mathematics and Computation, Federal University of Itajubá \\ Itajubá, Minas Gerais, 37500-903, Brazil \\ klippert@unifei.edu.br \\ VITORIO A. DE LORENCI \\ Department of Physics and Chemistry, Federal University of Itajubá \\ Itajubá, Minas Gerais, 37500-903, Brazil \\ delorenci@unifei.edu.br \\ SHI-YUAN LI \\ School of Physics, Shandong University \\ Shandong-Jinan, 250100, P.R.C. \\ lishy@sdu.edu.cn
}

Received 12 June 2011

Revised 29 June 2011

\begin{abstract}
The matter state appearing just after the inelastic hadronic collisions, presently called 'glasma', is here taken as the dominant matter-energy content of the early universe. As a result, an anisotropic cosmological toy-model is obtained in the limit of early times. This is a simple example of a collider physics motivated cosmology, the relevance of which relying in the occurrence of a true cosmic singularity (as usual big-bang models) despite the fact that the resulting metric tensor is regular everywhere.
\end{abstract}

Keywords: Glasma phenomenology; early Universe cosmology.

PACS numbers: $98.80 . \mathrm{Cq}, 04.40 .-\mathrm{b}$

\section{Introduction}

The universe is expanding. Hence, even in non-singular cosmological models, in early times it was more dense than it is today. The standard big-bang cosmology ${ }^{1-4}$ with classical Maxwell radiation as the matter content presents a singular behavior, ${ }^{5,6}$ identified as the origin of the universe. The spacetime curvature becomes arbitrarily large around the singular point, hence leaving room for arbitrarily large energetic processes as well as arbitrarily dense matter states to take place. The matter states of largest densities that human being can produce and study microscopically in laboratory are those produced in high energy collisions. It is then natural to discuss cosmology by evolving such states within the framework of gravity dynamics. In 
this paper, the cosmological consequences of considering a 'glasma' fluid extracted from collider physics as the dominant matter-energy content of the early universe are investigated.

There are many aspects in collider physics. We concentrate on the multi-particle system produced just after the inelastic and non-diffractive hadronic/nuclear collisions at high energies. Without restricting (triggering) the transverse momenta to be large, this multi-particle process is responsible for the increasing of the total cross section as the function of the collision energy, thus becoming dominant at very high energies. ${ }^{7}$ In relativistic heavy ion collisions, this kind of process is triggered and classified by the multiplicity of the produced hadrons. It is believed that the quarkgluon plasma is most probably produced for the largest multiplicity cases, as the evolution result from the multi-parton system, 'glasma', ${ }^{8-10}$ produced in the collision. Though the global distribution (e.g., longitudinal) is very similar in hadronic and nuclear collisions, for the latter case the produced glasma can be much more dense, with larger spacetime area, so that it may be taken as a 'medium', representing a kind of matter state. Hence, we choose this glasma system produced in the heavy ion collisions with the largest multiplicities (central collisions) as the basic object to extract the matter state of the early universe. The energy-momentum tensor $T^{\mu \nu}$ of the glasma ${ }^{9,10}$ is taken as the source fluid of the Einstein equation, which is then solved. When the details of transverse distribution are ignored, the form of the $T^{\mu \nu}$ is determined, which is similar to Bjorken hydrodynamics. ${ }^{11}$ The longitudinal distribution of the glasma system is rapidity-independent, with rapidity defined by $\eta=(1 / 2) \ln [(t+z) /(t-z)]$ in terms of Minkowskian-Cartesian coordinates $(t, x, y, z)$ with $z$ directed along the axis of the (nearly head-on) collision. This yields a $T^{\mu \nu}$ which depends only on proper time $\tau=\sqrt{t^{2}-z^{2}}$ in the co-moving frame, as described bellow. The formal structure of $T^{\mu \nu}$ at $z=0$ in the center-ofmass frame of the collision is the only ingredient we need, and we assume for it the form $T^{\mu \nu}=\operatorname{diag}\left(\varepsilon, p, p, p_{3}\right)$.

\section{The Model}

Let us look for solutions of the Einstein's field equations, $G^{\mu \nu}=\kappa T^{\mu \nu}$, with the special form

$$
\mathrm{d} s^{2}=\mathrm{d} \tau^{2}-\mathrm{A}^{2}(\tau)\left[\mathrm{d} x^{2}+\mathrm{d} y^{2}\right]-\mathrm{B}^{2}(\tau) \mathrm{d} \eta^{2},
$$

where $\mathrm{A}(\tau)$ and $\mathrm{B}(\tau)$ are functions to be determined. Einstein's field equations then yield a coupled set of nonlinear differential equations for $\mathrm{A}(\tau)$ and $\mathrm{B}(\tau)$ as

$$
\begin{aligned}
a^{2}+2 a b & =\kappa \varepsilon, \\
\dot{a}+\dot{b}+a b+a^{2}+b^{2} & =-\kappa \bar{p}, \\
2 \dot{a}+3 a^{2} & =-\kappa \bar{p}_{3},
\end{aligned}
$$

in terms of the new variables $a=\dot{\mathrm{A}} / \mathrm{A}, b=\dot{\mathrm{B}} / \mathrm{B}, \bar{p}=p \mathrm{~A}^{2}$ and $\bar{p}_{3}=p_{3} \mathrm{~B}^{2}$. 
Let us now examine a more specific example of the rapidity plateau regime, namely the phenomenological expression

$$
\varepsilon=12 \lambda Y^{2}
$$

for the energy density of the glasma, ${ }^{12}$ which is supposed to hold for $\tau \rightarrow 0^{+}$, where $\lambda$ is a constant and the time evolution is encoded in $Y=\ln \left(Q_{s} \tau\right)$ with $Q_{s}$ a constant being determined from QCD. We therefore limit our considerations of this model only in the limit of small positive $\tau$. In this sense, we put $B(\tau)=\tau[1+g(\tau)]$ as an Ansatz, where $|g(\tau)| \ll 1$.

Neglecting orders of $g$ higher than one, it follows that $b=1 / \tau+\dot{g}$, from which Eq. (2) can be algebraically solved as $a=6 \kappa \lambda \tau Y^{2}$. Inserting these results in Eqs. (3)-(4) we obtain $\bar{p}=-12 \lambda\left(Y^{2}-Y\right)$ and $\bar{p}_{3}=-12 \lambda\left(Y^{2}+2 Y\right)$, apart from finite terms as $\tau$ approach 0 . The small function $g$ is subjected to

$$
\ddot{g}+\frac{2}{\tau}\left[1-3 \kappa \lambda \tau^{2} Y^{2}-\frac{1}{Y}\right] \dot{g}=12 \kappa \lambda Y,
$$

the solution of which, finite as $\tau \rightarrow 0^{+}$, roughly behaves as $g(\tau) \simeq 2 \kappa \lambda \tau^{2} Y$. Since $|Y| \ll Y^{2}$, this solution is to describe an energy-momentum tensor of the form

$$
T_{\nu}^{\mu}=\varepsilon \delta_{\nu}^{\mu}
$$

from which the trace is $T=48 \lambda Y^{2}$. Eq. (7) makes it explicit that the dominant behavior of the glasma fluid is that of a running cosmological constant. The geometry takes the form given by the line element

$$
\mathrm{d} s^{2}=\mathrm{d} \tau^{2}-A_{0}^{2}\left(1+6 \kappa \lambda \tau^{2} Y^{2}\right)\left(\mathrm{d} x^{2}+\mathrm{d} y^{2}\right)-\tau^{2}\left(1+4 \kappa \lambda \tau^{2} Y\right) \mathrm{d} \eta^{2} .
$$

Thus, $A_{0}$ turns out to be an unspecified parameter which cannot be determined by the usual relation $\mathrm{A}$ (today) $=1$, since this model is not assumed to hold for large values of the $\tau$ coordinate. The solution Eq. (8) describes a spacetime whose axes expand at different rates. Although the metric tensor is regular at $\tau=0$, the spacetime displays a singular behavior at that instant as its Ricci scalar diverges. That is to say, physical singularities may be hidden in a finite metric tensor. This situation can be seen as the converse of the old discussion concerning the behavior of the horizon event in Schwarzschild geometry: in that case, the metric tensor (written in usual Schwarzschild coordinates) displays an apparent singularity at the horizon, but the spacetime is regular there as the manifold can be extended by GullstrandPainlevé, Lemaître, Eddington-Finkelstein, or Kruskal-Szekeres geometries.

Finally, it should be stressed that our results do not rely on the occurrence of a high energy collision in the early universe. Instead, we assume that the highly dense states of matter (such as the ones which occur in high energy collisions) was the dominant contribution at early stages of the universe. 


\section{Conclusion}

The induced cosmological model given from Eq. (8) is uniquely defined (up to $A_{0}$ ) because the energy density has been obtained from phenomenology. One should note, however, that the present model can only be used to describe a finite phase of the universe where the energy-momentum tensor proposed above is being considered to be the dominant contribution. This contribution is significative only for small times $\tau \sim 0$. In fact, as the glasma fluid evolves in time the energy-momentum dominance may also evolve to another form of matter, as a quark-gluon plasma ${ }^{13}$ or, later, classical radiation.

Another feature of the model above concerns the physical interpretation of time. It is apparent from the definition of $\tau$, and also from Eq. (8), that the 'coordinate' $\tau$ has the meaning of proper time (from the event of the collision up to the considered event in spacetime causally connected to the collision) as measured in the Minkowskian spacetime $\mathrm{d} s^{2}=\mathrm{d} t^{2}-\left(\mathrm{d} x^{2}+\mathrm{d} y^{2}+\mathrm{d} z^{2}\right)=\mathrm{d} \tau^{2}-\left(\mathrm{d} x^{2}+\mathrm{d} y^{2}+\tau^{2} \mathrm{~d} \eta^{2}\right)$. That is to say, the above definition of $\tau$ measures not the proper time in the evolving spacetime geometry in Eq. (8), but instead the proper time in flat Minkowski geometry. Would one seek the Euclidean section $(k=0)$ of the homogeneous and isotropic Friedmann-Lemaître-Robertson-Walker (FLRW) geometry

$$
\mathrm{d} s^{2}=\mathrm{d} t^{2}-[A(t)]^{2}\left(\mathrm{~d} r^{2}+r^{2}\left(\mathrm{~d} \theta^{2}+\sin ^{2} \theta \mathrm{d} \varphi^{2}\right)\right],
$$

in terms of its own proper time $\tau$, the result would then be found in the form

$$
\mathrm{d} s^{2}=\mathrm{d} \tau^{2}-2 k(\lambda) \mathrm{d} \tau \mathrm{d} \lambda-\mathrm{A}^{2}(\tau)\left[\mathrm{d} \lambda^{2}+r^{2}(\tau, \lambda)\left(\mathrm{d} \theta^{2}+\sin ^{2} \theta \mathrm{d} \phi^{2}\right)\right],
$$

where $\lambda$ measures the slope at the collision event of the causal geodesic curve which extends from the collision to the event of interest. The use of proper time as a Gaussian time coordinate of a non-static spacetime lacks a stationary representation of the geometry, as occurs in Eq. (10). There exists a constraint also, which correlate the functions $k(\lambda)$ and $A(\tau)$. The use of Eq. (10) as a suitable model of the glasma fluid given in Eq. (7) is a subject of current investigation.

\section{Acknowledgments}

This work is partially supported by CNPq and FAPEMIG (Brazil), National Natural Science Foundation of China (NSFC, grant Nos.10775090, 10935012) and Natural Science Foundation of Shandong Province, China (grant No. ZR2009AM001). S.-Y. L. thanks the hospitality of UNIFEI during his visit.

\section{References}

1. A. Friedmann, Zeitschr. Phys. 10, 377 (1922); 21, 326 (1924).

2. G. Lemaître, Ann. Soc. Sci. Bruxelles A 47, 29 (1927).

3. H. P. Robertson, Ap. J. 82, 248 (1935).

4. A. G. Walker, Proc. Lond. Math. Soc. 42, 90 (1936). 
5. S. W. Hawking and G. F. R. Ellis, The Large Scale Structure of Spacetime (Cambridge University Press, Cambridge, 1973); see also R. M. Wald, General Relativity (Univ. Chicago Press, Chicago, 1984).

6. E. W. Kolb and M. S. Turner, The Early Universe (Addison Wesley, San Francisko, 1990).

7. H. Cheng and T. T. Wu, Expanding Protons: Scattering at High Energies (MIT Press, Massachusetts, 1987).

8. T. Lappi and L. McLerran, Nucl. Phys. A 772, 200 (2006).

9. Y. V. Kovchegov, Nucl. Phys. A 762, 298 (2005).

10. Y. V. Kovchegov, Nucl. Phys. A 830, 395 (2009).

11. J. D. Bjorken, Phys. Rev. D 27, 140 (1983).

12. T. Lappi, Phys. Lett. B 643, 11 (2006); K. Fukushima, Phys. Rev. C 76, 021902(R) (2007); 77, 029901(E) (2007).

13. V. A. De Lorenci, Class. Quantum Grav. 27, 065007 (2010). 